\title{
Relato de caso - Capeamento pulpar direto em molar pós fratura de coroa
}

\author{
Case report - Direct pulp capping in molar post crown fracture
}

Recebido: 08/10/2021 | Revisado: 15/10/2021 | Aceito: 16/10/2021 | Publicado: 19/10/2021

Rafael Freitas de Arruda Universidade Brasil, Brasil

E-mail: rafael93freitas@gmail.com

Vinicius Quintas Gonçalves

Universidade Brasil, Brasil

E-mail: quintasvinicius34@gmail.com

José Lucas Martins

Universidade Brasil, Brasil

E-mail: lucas.martins@universidadebrasil.edu.br

\begin{abstract}
Resumo
O capeamento pulpar é um procedimento odontológico utilizado no cotidiano clínico, porém muito discutido na odontologia. Parte dos profissionais concordam que após a realização de forma consciente um bom diagnóstico pulpar, evitaria um tratamento endodôntico imediato. O estudo teve como finalidade a manutenção da vitalidade pulpar com através da realização do capeamento pulpar direto em um paciente adulto jovem, que apresentava queixa álgica no elemento 47. Ao exame radiográfico observou-se imagem radiolúcida sugestiva de lesão cariosa que se confirmou com o exame clínico, realizado o teste de vitalidade pulpar com resposta positiva foi realizado procedimento clínico restaurador e o acompanhamento por 40 dias com o objetivo de constatação do sucesso desejado, o que foi confirmado através dos testes clínicos e radiográficos.
\end{abstract}

Palavras-chave: Capeamento pulpar; Lesão cariosa; Materiais dentários; Saúde pulpar; Fratura de coroa.

\begin{abstract}
Pulp capping is a dental procedure used in daily clinical practice, but much discussed in dentistry. Some professionals agree that after consciously carrying out a good pulp diagnosis, immediate endodontic treatment would be avoided. The study had as important the maintenance of pulp vitality through direct pulp capping in a young adult patient, who presented pain in element 47 . The radiographic examination revealed a radiolucent image suggestive of a carious lesion, which was confirmed by the examination clinical, performed the pulp vitality test with a positive response, a restorative clinical procedure and follow-up for 40 days were performed with the objective of verifying the desired success, which was confirmed through clinical and radiographic tests.
\end{abstract}

Keywords: Pulp capping; Carious lesion; Dental materials; Soft pulp; Crown fracture.

\section{Introdução}

A saúde de um modo geral é um bem imaterial e indispensável para a vida humana, está diretamente relacionada às condições de alimentação, moradia, trabalho, renda, meio ambiente, transporte, lazer, acesso à serviços de saúde e informação. Nesse sentido a luta pela saúde bucal, está fundamentalmente ligada à luta pela melhoria dos determinantes sociais, políticos e econômicos. (PAULETO A.R.C et al, 2004).

Narvai e Frazão, 2005 acrescentam ainda em relação a saúde bucal, que devemos considerar um conjunto de condições sendo objetivas (biológicas) e subjetivas (psicológicas), que permitem o ser humano exercer várias funções, desenvolver a autoestima e relacionar-se socialmente sem inibição ou constrangimento.

A proteção do complexo dentino-pulpar há décadas vem sendo discutido e questionado pelos profissionais cirurgiões-dentistas. Entretanto, é indiscutível nos dias atuais que o tratamento conservador da polpa é sempre a melhor opção tanto para o paciente quanto para o profissional, e diante do aumento do número de casos de dentes cariados e fraturados, tanto em adultos quanto em crianças essa opção deve ser considerada facilitando o processo restaurador. (SANTOS, OLIVEIRA, 2019). 
Os tratamentos ao órgão dental visando a manutenção da vitalidade pulpar podem ser divididos em capeamento pulpar direto e capeamento pulpar indireto. (CARVALHO et al, 2012, DAMASHEK, GALLER, KRASTEL, 2019). O tratamento conservador da polpa, hoje denominado terapia vital da polpa, busca preservar a vitalidade pulpar isolando a dentina exposta e a polpa subjacente de estímulos externos, como microrganismos e os componentes tóxicos dos materiais restauradores. Além de formar uma barreira mineralizada capaz de recuperar o tecido pulpar, garantindo a manutenção de suas funções, preservando o elemento dentário na cavidade bucal com vitalidade.

Há diversos estudos em relação aos materiais capeadores de escolha para realização da proteção pulpar, mas os objetivos são comuns, entre eles estão o combate às bactérias, impedir a progressão da cárie, estimular as células da polpa, formar nova dentina e produzir um selamento durável, que protege o complexo pulpar (ALEX,2018).

KOBAYASHI et al (2016) afirma que a taxa de sucesso do capeamento pulpar direto é inferior ao da pulpectomia e que os materiais atuais não têm eficácia, principalmente por causa do selamento na dentina, bem como do material restaurador, sugerindo a utilização de materiais adesivos para melhorar a taxa de sucesso a longo prazo.

ROSA et al ( 2017) apontam o hidróxido de cálcio como o principal tipo de material estudado, principalmente para capeamento direto da polpa; avanços recentes em materiais bioativos mostram resultados promissores que poderiam melhorar os biomateriais utilizados nos tratamentos vitais da polpa, entre eles podemos citar o Agregado Trióxido Mineral (MTA) e a resina MMA-TBB como um capeador direto no futuro, KOBAYASHI et al (2016); que apresenta ótimos resultados defendendo que este material criaria uma ponte artificial sobre a polpa imediatamente, enquanto os materiais bioativos levam pelo menos uma semana.

O presente relato de caso clínico tem como objetivo apresentar a técnica de capeamento pulpar como uma terapia positiva para manutenção da vitalidade da polpa, através de Capeamento Direto em paciente jovem com acometimento da doença cárie e com fratura coronal.

\section{Metodologia}

Este artigo trata-se de um relato de caso, abordado de forma descritiva e qualitativa, onde segundo Pereira et al. (2018), caracteriza-se como uma pesquisa que por via direta recolhe dados relativos ao estudo.

\section{Relato de Caso}

Paciente jovem do gênero masculino com idade de 22 anos compareceu a clínica da Universidade Brasil queixando do elemento 47 ( $2^{\circ}$ molar inferior). Relatava sensibilidade ao contato com o frio e dor espontânea que passava rapidamente. O paciente relatou também que já havia sido restaurado duas vezes em outra clínica odontológica, mas não obteve resultado satisfatório. Realizamos os exames radiográficos e clínicos que incluíram o teste térmico com o gás refrigerante e percussão vertical, ambos apresentaram respostas positivas aos testes.

A seguir observa-se a Figura 1, no qual representa o estado inicial do elemento dentário. 
Figura 1 - Elemento 47 fraturado.

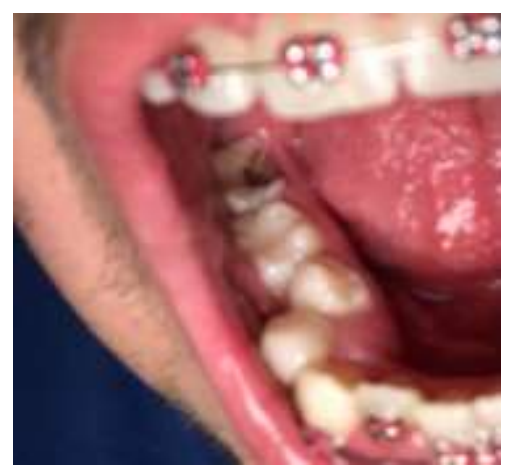

Fonte: Rafael Freitas (23-03-2021).

Na radiografia identificamos que o elemento estava comprometido com lesão de cárie e uma infiltração, observado por imagem radiolúcida bem detalhada, abaixo da restauração em resina se aproximando a câmera pulpar (Figura 2).

Figura 2 - Radiografia com infiltração e lesão de cárie.

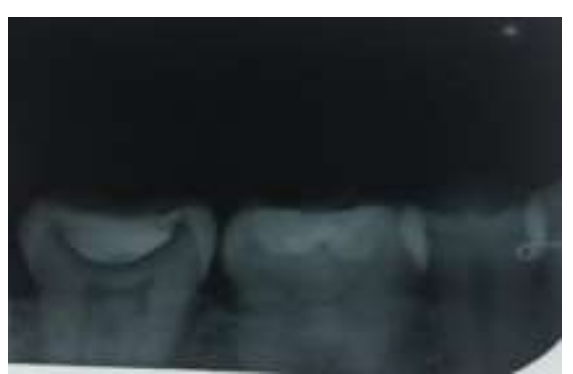

Fonte: Rafael Freitas (23-03-2021).

Após avaliação e ponderação dos resultados começamos o preparo inicial da cavidade. O paciente foi anestesiado com bloqueio regional do nervo alveolar inferior (anestésico de escolha: lidocaína 2\%). Foi realizada a remoção do restante da restauração antiga que permanecia no elemento, utilizando caneta de alta rotação com brocas esféricas Carbide obtemos acesso à lesão de cárie. Não tivemos dificuldade nessa etapa do procedimento clínico. Quando removida totalmente a restauração, identificamos uma lesão de cárie ativa em dentina (score 5) com um aspecto de tecido amolecido e marrom claro. Segue a Figura 3, representando o preparo cavitário.

Figura 3 - Preparo cavitário inicial.

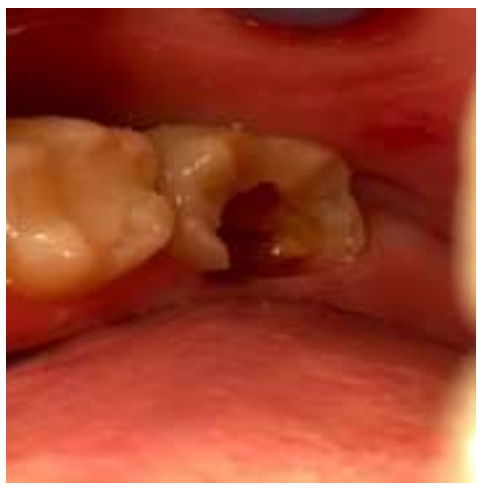

Fonte: Rafael Freitas (23-03-2021).

Na Figura 4 pode-se observar a remoção da dentina afetada. 
Figura 4 - Remoção da dentina.

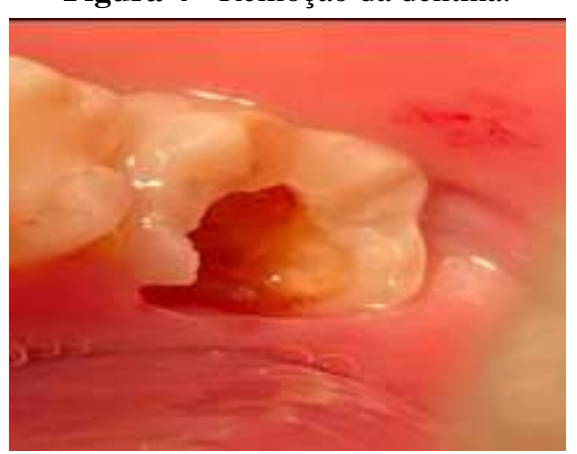

Fonte: Brenda Flores (23-03-2021)

Para realizar essa etapa do procedimento utilizamos instrumentos manuais, como colher de dentina que ajudou na remoção do tecido cariado e instrumento rotatório (caneta de baixa rotação) utilizando broca esférica, com muito cuidado para não atingir a câmara pulpar. Foi feita a remoção total da cárie em todas as paredes circundantes e a parede pulpar.

Figura 5 a seguir mostra a micro exposição pulpar. No final do procedimento ocorreu uma micro exposição pulpar e com isso tivemos que usar a técnica capeamento pulpar direito. Fizemos a lavagem da cavidade soro fisiológico e secamos para conseguirmos ter uma boa forma de conveniência, usando alguns materiais protetores do complexo dentinopulpar como: cimento de hidróxido de cálcio P.A no local da exposição e ionômero de vidro como material restaurador provisório.

Figura 5: Cavidade com exposição pulpar.

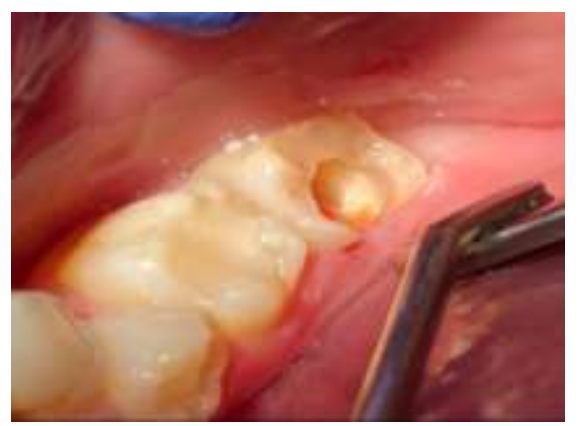

Fonte: Rafael Freitas (23-03-2021).

Após aplicações dos materiais de proteção do complexo dentinopulpar, finalizamos com a checagem da oclusão do paciente, receitamos um medicamento pós operatório (dipirona em gotas - 30 gotas por via oral, a cada 6 horas por 3 dias. Marcamos retorno após 40 dias, para reavaliação do procedimento.

O paciente retornou à clínica da universidade Brasil, após 40 dias. Relata que seguiu orientações e prescrições pósoperatórias. Não havendo mais sensibilidade ao contato com o frio e nem dor. Observa-se na Figura 6 a radiografia de retorno do paciente.

Figura 6 - Radiografia de proservação (40 dias).

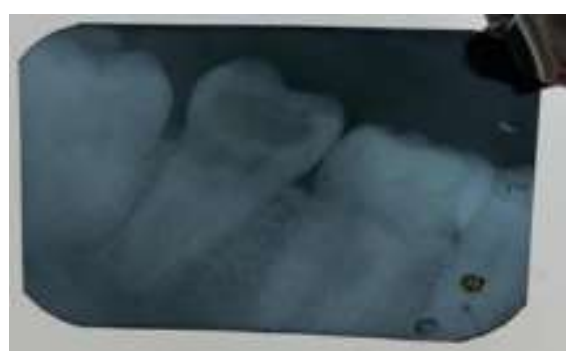

Fonte: Vinicius Quintas (25-05-2021) 
Observamos que o tratamento de capeamento pulpar direito, na medida do possível, foi bem sucedido. O paciente não queixa-se mais de dor e sensibilidade, a lesão de cárie foi removida e por hora controlada.

Em seguida removemos uma parte muito superficial do ionômero de vidro, utilizamos o sistema adesivo convencional de 2 passos: ácido fosfórico a 37\% e o adesivo (Primer e Bond). Finalizamos a restauração com resina composta (Figura 7).

Figura 7 - Elemento restaurado com resina composta.

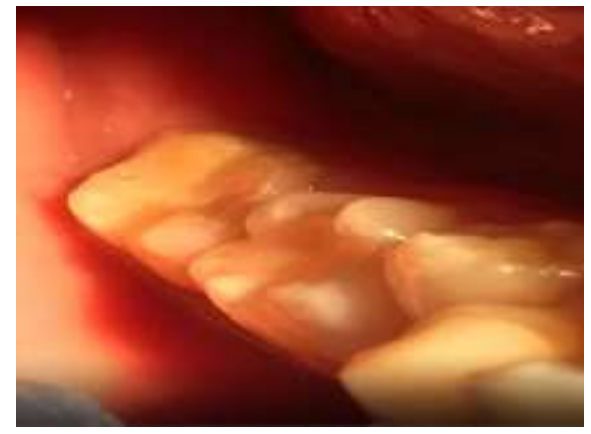

Fonte: Brenda Flores (25-05-2021).

O elemento foi restaurado com resina composta usando técnica incremental e fotopolimerizado. Nesse caso clínico, tivemos um bom sucesso no tratamento de capeamento pulpar direto, conseguindo resgatar a saúde bucal, função e estética para o paciente novamente.

\section{Discussão}

De maneira simplificada o capeamento pulpar é classificado como tratamento conservador ao órgão pulpar, impedindo o seu comprometimento. A remoção da cárie, mesmo de forma parcial, impedindo a exposição pulpar é o maior objetivo. Porém, quando ocorre a exposição da polpa, seja ela devido à lesão cariosa ou até mesmo por fratura coronária o procedimento torna-se mais complexo porém o objetivo continua sendo o mesmo, preservar a vitalidade pulpar.

Segundo Carvalho et al. (2012) a idade do paciente é apontada como de grande influência, pois a resposta biológica pulpar vai se tornando mais lenta e reduzida com o passar dos anos, então tratamentos conservadores são mais indicados para pacientes jovens.

Os materiais capeadores pulpares são aplicados diretamente sobre a superfície da polpa exposta com a finalidade de protegê-la contra injúrias químicas, físicas e biológicas, permitindo a formação de dentina reparadora. (PRECZEVSKI, 2016)

Apresenta como desvantagens não ter adesão à dentina, afeta a polimerização de materiais restauradores resinosos, tem alta solubilidade e baixa resistência mecânica. Apesar de estimular a formação de dentina reparadora causa necrose quando em contato com o tecido pulpar devido a sua alta alcalinidade, o que pode levar as respostas inflamatórias pulpares. (AKHAVAN et al. 2017, ROSA et al, 2017; DAMASCHKE, GALLER, KRASTL, 2019).

Os sistemas adesivos sempre apresentam uma resposta inflamatória na polpa, que num primeiro momento tem características agudas e se mantém por um período prolongado com características crônicas, além de apresentar necrose tecidual e quando há formação de tecido duro, este se apresenta em uma pequena espessura, além de não ser possível observar a formação de uma nova camada odontoblástica. Enquanto que o hidróxido de cálcio e os cimentos de silicato de cálcio, quando utilizados apresentam menor inflamação, formação de camada odontoblástica e de barreira de tecido duro com maior espessura (MASCIOLI et al.2007, ARAÚJO et al. 2011, NOVICKA et al. 2016, AKHAVAN et al 2017, KAMAL et al. 2018). 
No caso clínico relatado, o material de escolha para proteção ao complexo-dentino pulpar foi Hidroxido de Calcio P.A, pois ocorreu acidentalmente uma micro exposição pulpar, ficando inviável utilizar qualquer tipo de sistema adesivo, resultando uma irritação na polpa.

Os insucessos da terapia de capeamento pulpar direto ocorrem, sobretudo, pela presença de contaminação na interface dente-material restaurador. Deve-se realizar um acompanhamento clínico, avaliando o teste de vitalidade e exame radiográfico em intervalos de 3,6 e 12 meses para atestar o sucesso clínico do procedimento. Como resultado do procedimento pode haver calcificações pulpares, que atingem $40 \%$ em até 10 anos. Os casos de exposição por traumatismo dentário têm uma taxa de sucesso que varia de 54 a 90\% e o material influencia neste sucesso tendo o hidróxido de cálcio uma taxa de 60\%. (SANTOS, OLIVEIRA, 2019).

No tratamento realizado de capeamento pulpar direto com Hidróxido de Cálcio P.A, orientamos o paciente a retornar no período de 40 dias, seguindo assim a recomendação da literatura. Foi observado clinicamente e com auxílio de exames complementares, que o procedimento atingiu uma taxa favorável de sucesso.

Segundo os autores Dammascke 2019, Galler 2019, Krastl 2019, os materiais a base de hidróxido cálcio são de primeira escolha para o tratamento de capeamento pulpar, pois liberam a substância por um longo período, tendo ação antimicrobiana prolongada. Entretanto, um dos fatores contra é que podem causar descoloração nos dentes, sobretudo quando possuem ferro na sua composição.

Após revisão da literatura baseando-se nos autores citados acima, destacamos alguns levantamentos pertinentes aos pòs e contras ao capeamento pulpar direto e seus devidos materiais utilizados como protetores. Alguns autores defendem o tratamento com o uso de hidróxido de cálcio por conta do estímulo da dentina reparadora e eficácia do procedimento nos pacientes jovens/adultos. Por outro lado, há controvérsias no qual o material protetor pode ocasionar descoloração no elemento dental, principalmente se na sua composição tiver componentes à base de ferro.

\section{Conclusão}

No relato de caso descrito consideramos as seguintes questões:

A lesão de cárie apresentada foi classificada como profunda e extensa, com isso tivemos grande dificuldade em realizarmos um preparo mais conservador.

Nos dias atuais, há diversos materiais no mercado, porém o mais indicado e utilizado é o hidróxido de cálcio P.A, principalmente nas cavidades muito profundas e com exposição pulpar. Devido a micro exposição pulpar, decidimos utilizar o hidróxido de cálcio, realizando o tratamento de capeamento pulpar direto.

O objetivo do trabalho foi alcançado, pois o tratamento apresentou resultado positivo, restabelecendo a função mastigatória e controlando a algia.

Por fim ressaltamos a importância do acompanhamento intensivo do paciente, durante pelo menos 12 meses, realizando tomadas radiográficas para qualquer sinal de descompensação, avaliarmos a possibilidade de um tratamento endodôntico.

Para continuidade do presente trabalho, deixamos como sugestão a realização do capeamento pulpar indireto, sendo este mais conservador, evitando assim a exposição pulpar e também a intervenção endodôntica.

\section{Referências}

AGNES, A., LONG, A., BEST, S. \& LOBNER, D. Pulp capping materials alter the toxicity and oxidative stress induced by composite resins in dental pulp culture. Journal European endodontic, v. 2. p. 2-6. 2017.

AKHAVAN, A., ARBABZADEH, F., BOUZARI, M., RAZAVI, S. M. \& DAVOUDI, A. Pulp response following direct pulp capping with dentin adhesives and mineral trioxide aggregate: an animal study. Journal Iranian endodontic. v.12, n.2, p. 226-30. 2017.

ALEX, G. Direct and indirect pulp capping: a brief history, material innovations and clinical case report. Compendium. v.39, n.3, p.182-189. 2018 
ARAÚJO F J, BARBOSA S J C, MOYSÉS M R, PEREIRA A A C, RIBEIRO J C R, RIBEIRO J G R, MENDONÇA A T. Análise histológica da biocompatibilidade de adesivos dentinários. Rev. da Universidade Vale do Rio Verde v.9, n.2, p.247-254. 2011.

CARVALHO C N, FREIRE L G, NAKAMURA V, GAVANI G. Possibilidades terapêuticas no tratamento de dentes jovens portadores de polpa viva: uma revisão da literatura. Rev. Ciência. Saúde v.14, n.1, p.40-52. 2012.

CAVALCANTI B N B., ARSCHNEIDER L R, MARQUES M M. Cytotoxicity of substances leached from a conventional and a self-etching adhesive system on human pulp fibroblastos. Braz dent sci v.13, n.2, p.10-14.42. 2010.

CHEN L, SUH B I. Cytotoxicity and biocompatibility of resin-free and resin-modified direct pulp capping materials: a state-of-the-art review. Dental materials journal v.36 n.1, p.1-7. 2017.

DAMMASCHKE T, GALLER K, KRAST L G. Current recommendations for vital pulp treatment. Rev. Deutsche Zahnärztliche Zeitschrift International. v.1, n.1, p.44-52. 2019.

DA SILVA T M, DA ROSA W L O, MARQUES M R, et al. Does Laser Improve The Clinical Success In Direct Pulp Capping? A Systematic Review And Meta-Analysis. J Oralhealth Dent Care.v.1 n.4. 2017.

FRAZÃO P, NARVAI P C. Saúde bucal no Sistema Único de Saúde: 20 anos de lutas por uma política pública. Rev Saúde em Debate, Rio de Janeiro. v. 33, n.81, p.64-71, jan.labr. 2005.

KOBAYASHI T, ZHU Q, EBERHART R, IMAI Y. Current status of direct pulp-capping materials for permanent teeth. Dental Materials Journal , v.35(1), n.1, p.12. 2016.

MASIOLI M A, DIAS K R C, VARGAS P R I, ROSA R. Avaliação microscópica de polpas humanas expostas em contato direto com adesivo dentinário e hidróxido de cálcio p.a. UFES REV. ODONTOL v.9, n.3, p.4-11. 2007.

MODERNA K C DA S, CASAS-APAYCO L C, ATTA M T, COSTA C A DE S, HEBLING J, SIPERT C R, NAVARRO M F DE L, SANTOS C F. Cytotoxicity and biocompatibility of direct and indirect pulp capping materials. J APPL ORAL SCI. v.17, n.6, p.54. 2009.

PEREIRA A S et al. Metodologia da pesquisa científica. Santa Maria. ED. UAB/NTE/UFSM 2018.

ROSA R. Avaliação microscópica de polpas humanas expostas em contato direto com adesivo dentinário e hidróxido de cálcio p.a. UFES REV. ODONTOL. v.9, n.3, p.4-11. 2007.

ROSA W L O, COCCO A R, SILVA, T M, MESQUITA L C, GALARC A D, SILVA A F, MASIOLI E P, DIAS K R H C, VARGAS P R M. Current trends and future perspectives of dental pulp capping materials: a systematic review. Journal Of Biomedical Materials Research 